\title{
Utilización de un dispositivo electrónico de última generación para la determinación experimental del coeficiente de convección de una placa plana de aluminio
}

Use of last generation electronic device for the experimental computation of the convection coefficient over a flat aluminum plate

Luis Diego Ramírez-González ${ }^{1}$, Luis Parras-Anguita², Carlos del Pino-Peñas ${ }^{3}$

Fecha de recepción: 21 de junio de 2018

Fecha de aprobación: 13 de setiembre de 2018

Ramírez-González, L D; Parras-Anguita, L; Pino-Peñas, C. Utilización de un dispositivo electrónico de última generación para la determinación experimental del coeficiente de convección de una placa plana de aluminio. Tecnología en Marcha. Vol. 32-2. Abril-Junio 2019. Pág 122-133.

DOI: https://doi.org/10.18845/tm.v32i2.4354

Estudiante Ing. Electromecánica con énfasis en Mantenimiento Industrial, Instituto Tecnológico de Costa Rica. Costa Rica. Correo electrónico: Iramirez@ieee.org

2 PhD. Mecánica de fluidos. Profesor contratado Doctor e investigador, Universidad de Málaga (UMA), España. Correo electrónico: Iparras@uma.es

3 PhD. en Mecánica de fluidos. Profesor titular contratado doctor, investigador, Subdirector de Posgrado e Investigación Universidad de Málaga (UMA), España. Correo electrónico: cpino@uma.es 


\title{
Palabras clave
}

Dispositivo electrónico de última generación; transferencia de calor; coeficiente de convección.

\section{Resumen}

Este artículo considera la comprobación de funcionamiento de una matriz de sensores capaces de medir la temperatura, de un dispositivo diseñado y creado por el departamento de ingeniería electrónica de la Universidad de Málaga, España. Para las pruebas se utiliza un chorro de aire a una temperatura menor al ambiente, que impacta una placa plana de aluminio ubicada sobre dichos sensores. Modificando la relación adimensional entre la altura y el diámetro de salida del chorro $(H / D)$ y con un adecuado procesamiento de datos mediante el uso de Matlab se encuentran diferentes curvas características. Además, se determina el coeficiente de convección mediante la relación entre la pendiente de la gráfica resultante de la evolución temporal de la temperatura y las propiedades físicas del aluminio.

El objetivo principal del trabajo es la comprobación de la funcionalidad del dispositivo electrónico creado por la Universidad, el cual se realiza con éxito. Además, se utilizan técnicas de visualización para comprobar el comportamiento del chorro de aire fresco al impactar la placa. Tanto el dispositivo, como la utilización de chorros de aire tienen amplias funcionalidades en la industria (refrigeración, calefacción, procesos de secado, limpieza, entre otros) y algunas más específicas para el dispositivo como la medición a tiempo real de temperaturas bidimensionales en zonas de difícil acceso.

\section{Keywords}

Last generation electronic device; heat transfer; convection coefficient.

\begin{abstract}
This article deals with a jet of air at a lower temperature than the environment, which discharges onto a flat aluminum plate located over a matrix of sensors that measures the temperature in the area of impact. Subsequently, characteristic curves are found for each height and diameter ratio of the jet $(H / D)$, through data processing. The coefficient of convection is determined using the relationship between the slope of the graph together with the result from the temporal evolution of the temperature.

The main objective of the work is the verification of the functionality of the advanced electronic device, which is carried out successfully. Also, and using visualization techniques the behavior of the jet is analyzed. Both the electronic device and the use of air jets have great interest in the industry (refrigeration, heating, drying processes, cleaning among others). In the case of the advance electronic device it is possible to provide data regarding real-time temperature measurement in areas of difficult access.
\end{abstract}




\section{Introducción}

El impacto de chorros de diferentes fluidos, principalmente de agua o aire, tiene gran importancia en aplicaciones industriales, normalmente se utilizan chorros turbulentos debido a su capacidad calorífica. Sin embargo, en casos específicos se llegan a utilizar flujos en régimen laminar [1]. Se tienen diferentes ejemplos de chorros impactando sobre diferentes superficies. Hay aplicaciones en las que chorros impactan a gran escala sobre el suelo como los utilizados en el avión VTOL [2]. También hay unos a menor escala como los utilizados en la obtención de la fuerza cortante que sufre el tejido vascular en el estudio de la aterogénesis [3]. La utilización de chorros laminares está limitada para aspectos más específicos, ya que la relación $H / D(H$ distancia entre la superficie de impacto y el chorro, D el diámetro de salida del chorro) debe oscilar en valores bajos, por lo que montajes grandes o de alta velocidad normalmente no lo permiten [4].

Los primeros experimentos realizado en este tema datan de 1964, en el cual se hace un estudio para chorros laminares y turbulentos, los resultados son vigentes aún para nuestros experimentos. Estos primeros estudios se limitaron para un chorro perpendicular a la placa y sin variaciones de temperatura, posteriormente se realizaron más estudios para diferentes ángulos de impacto.

En el experimento realizado en la Universidad de Málaga (UMA) se recrea una experiencia similar a la mencionada anteriormente, un chorro de aire fresco que impacta perpendicularmente sobre una placa de aluminio que se encuentra a mayor temperatura. Se analiza este caso con un gradiente de temperatura con respecto a la ambiental por su interés industrial. Para la medición de la temperatura se utiliza un dispositivo electrónico diseñado y ensamblado en la Escuela de Ingenierías Industriales de la UMA que permite calcular cuantitativamente el cambio de temperatura que se produce a lo largo de la placa.

Según los desarrolladores del dispositivo (departamento de Ingeniería Electrónica de la UMA), este permite mediciones altamente precisas sin ser intrusivo y con una filosofía de tecnología electrónica que aumenta la lectura de múltiples sensores con un canal de datos minimizados. Además, es una solución para condiciones de medición complicadas, tal y como lo mencionan los creadores de la placa, por ejemplo, medir la temperatura de un asiento en un vehículo cuando hay una persona sentada, entre otros casos [5]. Existen otros tipos de placas para la medición de temperatura en arreglos similares a la utilizada en el experimento. Sin embargo, la electrónica utilizada hace de este dispositivo la mejor opción, ya que las otras placas similares para 64 sensores utilizan 128 cables y hacen la toma de datos en aproximadamente un minuto [6], mientras que el dispositivo utilizado tiene un arreglo de sensores que lo hace ser muy eficiente, utiliza pocos cables (3 cables de datos, 3 de alimentación) y la toma de datos se realiza en un tiempo del orden de milisegundos, todo esto a un coste bajo [5]. Cabe mencionar que el objetivo de este artículo es la comprobación del funcionamiento de la placa, más allá que su desarrollo, esta etapa estuvo a cargo del departamento de Ingeniería Electrónica de la UMA.

La forma de visualización más utilizada en experimentos con aire se basa en las partículas trazadoras de la técnica PIV (Particle Image Velocimetry), que está diseñada para determinar el campo instantáneo de velocidades en el plano del fluido. En este experimento la introducción de partículas visibles se realizó con un burbujeador de aceite dando lugar a una emulsión de aceite y aire fresco con un tamaño de sus partículas del orden de un micrómetro y con una densidad similar a la del aire. Estos dos aspectos hacen que la técnica sea no intrusiva, aspecto a considerar cuando se esté realizando mediciones con cualquier técnica de visualización. Si bien es cierto que el objetivo principal del PIV no es la observación cualitativa, al hacer incidir un plano láser sobre el fluido con las pequeñas partículas de aceite permite la visualización de las mismas, y así comprobar que para los parámetros utilizados (relaciones H/D, la temperatura 
de salida y de la placa, caudal, presión del ambiente) el fluido es laminar, inestable o turbulento antes y después del impacto [1].

Siendo capaces de realizar mediciones de temperatura a lo largo de la placa en tiempo real se puede realizar el estudio de la transferencia de calor que ocurre cuando la superficie sufre el impacto del chorro. La termodinámica reconoce como métodos de transferencia de calor: conducción, convección y radiación. En el caso de radiación (método en el cual se da la transferencia de calor desde el Sol hasta la Tierra), las partículas transfieren el calor a otras antes de chocar. Estos mecanismos de transferencia son más eficientes en ambientes vacíos y con pocos obstáculos. Por este motivo, en algunos casos se puede obviar su aporte ya que es insignificantico, ingenierilmente, con respecto a la transferencia por convección o conducción [8].

La convección, estrictamente hablando, es un transporte de energía por el movimiento en el medio. En ingeniería se suele asociar el término a la transferencia de calor que se da entre un fluido en movimiento y un cuerpo sólido. Existen dos tipos de convección, forzada y natural. La primera se refiere a un flujo que se destina para este fin, ya sea mediante un sistema de bombeo o impulsión, gravedad o procesos externos, mientras que la segunda se da por diferencias de densidades. Como se comentaba anteriormente, los flujos más utilizados en ingeniería para la transferencia de calor son turbulentos. En este régimen la convección forzada se suele aprovechar porque utilizan números de Reynolds elevados. No obstante, los fluidos laminares también son funcionales, según sea la aplicación [9].

Para casos de fluidos laminares principalmente (también pasa con flujos turbulentos, pero en menor medida), se da la condición de no deslizamiento mediante la cual el fluido se adhiere a una superficie no porosa, como una placa de aluminio y se convierte en una película sobre el sólido. Se dice que en fluidos turbulentos se da en menor medida por la recirculación del fluido. En la figura 1, Cengel ejemplifica el perfil de velocidades, los tamaños de las películas y las direcciones del fluido. En el experimento de este artículo se utilizó un fluido laminar, lo que corresponde a la primera parte del diagrama presentado en la figura 1. Se puede observar que la velocidad en la zona cercana a la placa es cero y en las zonas más alejadas es más alta, mientras que en fluidos turbulentos las velocidades lineales son menores, pero las capas más cercanas a la placa tienen más velocidad que en el caso laminar.

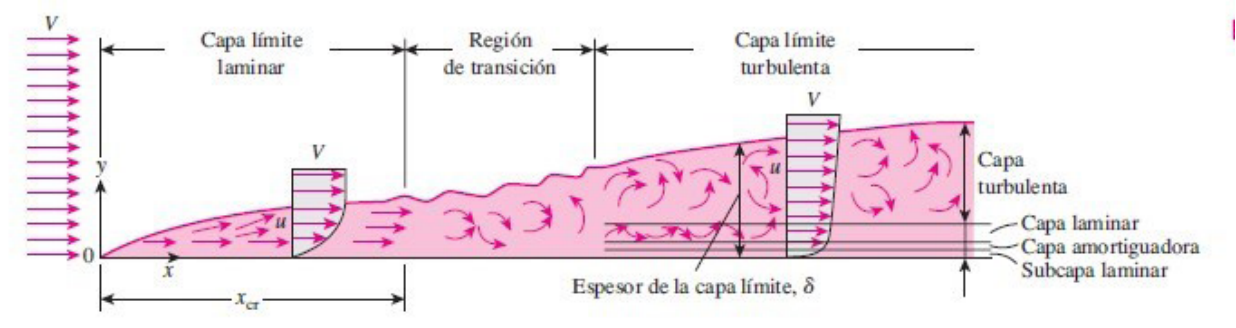

Figura 1. Desarrollo de la capa limite en una placa plana. [9]

La medición del efecto de película en muchos casos se convierte en una tarea complicada, por lo que obtener valores de coeficientes de convección es una tarea difícil. Se han obtenido rangos o valores típicos, como los presentados en el libro de Transferencia de Calor [9] para casos genéricos en donde se habla de un sólido impermeable y un gas o un líquido mediante convección libre o forzada. Para la combinación de convección libre, gas-sólido, se da un rango de [2-25] W/m2 K, para convección libre, líquido-sólido el rango es de [50-1000] W/m2 
K, para convección forzada, gas-sólido, se da un rango de [2-250] W/m2 K, para convección forzada, líquido-sólido el rango es de [50-2000] W/m2 K. El caso del experimento corresponde a convección forzada, en una interacción de gas-sólido.

En caso de no existir el efecto película, o que no tenga una aparición significativa, las mediciones deben de considerarse con una fricción cero. En este caso tanto la capa superior como inferior en un fluido laminar tienen la misma velocidad [10]. Esto quiere decir que, para el experimento realizado en este artículo, en caso de que no se haga presente el efecto de la viscosidad, los contornos de velocidad deben ser concéntricos y la temperatura aumenta desde el centro de la placa hasta que llegue a equipararse con la temperatura ambiente.

\section{Materiales y métodos}

El montaje experimental se realiza en la Universidad de Málaga, España. Se utilizó un cilindro de metacrilato con un diámetro de $400 \mathrm{~mm}$, y $1000 \mathrm{~mm}$ de alto esto con el fin de aislar y proteger de perturbaciones al chorro de aire, ya que éste puede verse influenciado por las corrientes de aire del laboratorio. Dentro del cilindro se ha colocado una mesa de elevación para la placa, con la finalidad de que el aire frío baje, se desplace por la parte inferior del cilindro y no interfiera con las mediciones del dispositivo. En la figura 2 se puede observar una fotografía del montaje experimental.

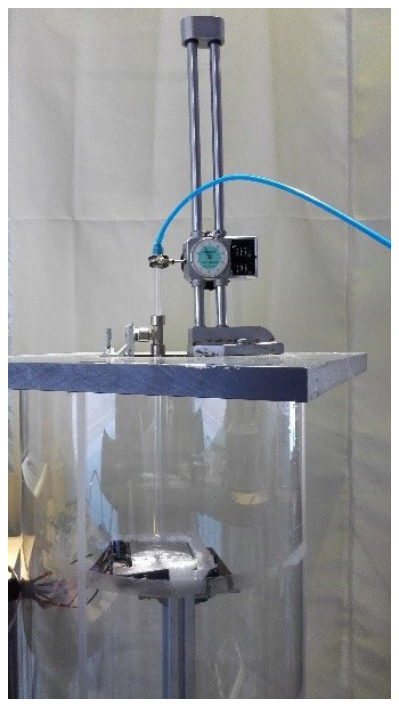

Figura 2. Montaje experimental.

En la parte superior del cilindro se coloca una tapa por donde se introduce el aire frío a una temperatura (T1) menor a la ambiental dentro del cilindro (T2). Para la inyección del fluido es necesario utilizar un compresor. Desde la salida del tanque de almacenamiento hasta el conducto de salida, la línea neumática debe pasar por un proceso de secado y enfriamiento. Es importante colocar el enfriador luego del secado porque al retirarle humedad la temperatura del fluido disminuye.

El tubo de salida tiene un diámetro interior de $6.87 \mathrm{~mm}$ y se utiliza un micrómetro de la marca Mitutoyo, el cual se presenta en la figura 3 (en conjunto con el soporte del tubo), para garantizar una relación $\mathrm{H} / \mathrm{D}$ bastante precisa, ya que se puede modificar el valor de $\mathrm{H}$ con una tolerancia de la centésima de milímetro. La zona de impacto es una superficie cuadrada de 140 x140 mm2, el diámetro del círculo circunscrito en el área de impacto es 20 veces mayor que el diámetro de salida del chorro, por lo que su relación es suficientemente grande para realizar el estudio deseado. 


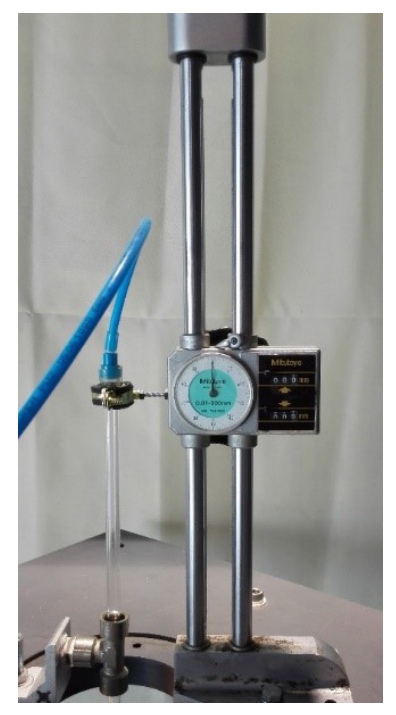

Figura 3. Micrómetro marca Mitutoyo. [9]

En el montaje se debe asegurar la perpendicularidad del chorro con respecto a la placa, además el nivelado de la placa. Para este fin, se utilizó la ayuda de un nivel digital de $\pm 0.1^{\circ}$ de precisión y $\pm 0.2^{\circ}$ de exactitud.

Para comprobar que el montaje permite flujos laminares se utiliza la ayuda de un láser continuo verde de $532 \mathrm{~nm}$ con una potencia máxima de $2 \mathrm{~W}$. El láser se hace pasar por un juego de lentes para abrir en un plano bidimensional, el cual debe incidir con el centro del tubo en la salida del chorro y se hacen observaciones cualitativas, en las cuales fue necesario la utilización de un burbujeador de aceite, el cual puede generar tanto humo que crea una mezcla homogénea sin cambiar la densidad del aire. Además, las partículas no son tóxicas por lo que no requiere equipo de seguridad. El burbujeador se presenta en la figura 4, en donde se puede observar el aceite vegetal, sin estar burbujeando, un tubo de PVC en el recipiente cerrado, la entrada y salida de aire con sus respectivas válvulas. La producción de burbujeo es estrictamente proporcional a la cantidad de aire inyectado, además es un productor de partículas continuo lo que facilita las visualizaciones.

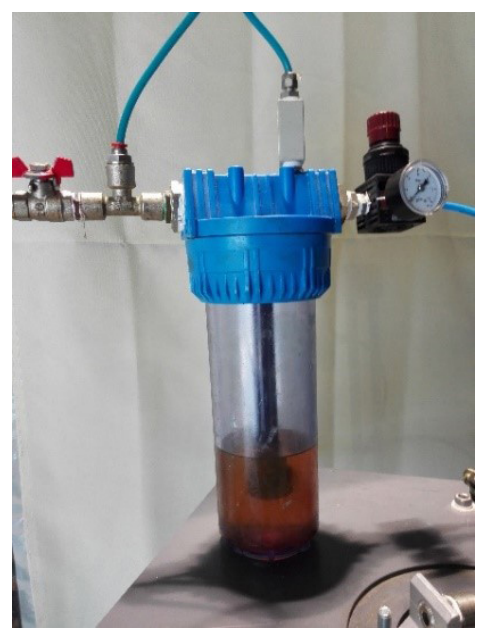

Figura 4. Burbujeador de aceite. 
El montaje del dispositivo de medición se puede observar en la figura 5. Como se pretende medir la temperatura y con la idea de aislarlo aún más del entorno se coloca corcho blanco en su perímetro. El cableado de alimentación y datos sale por un orificio ubicado en cilindro. Fuera del cilindro se tiene un ordenador para la recolección de datos de la placa de sensores que son traducidos por una placa Nexys 3, además una fuente de alimentación de $\pm 5 \mathrm{~V}$.

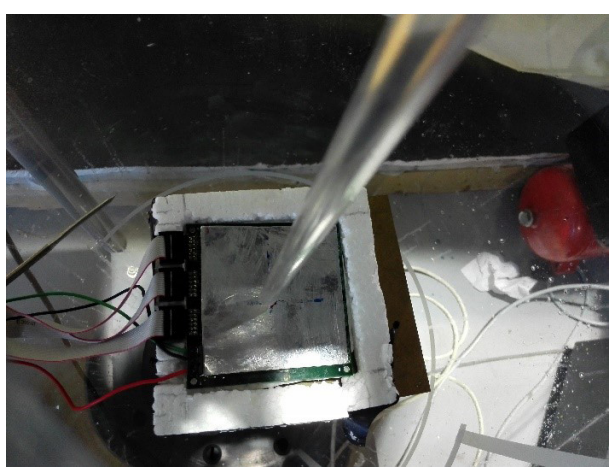

Figura 5. Montaje del dispositivo de medición.

Con el fin de tener una diferencia de temperaturas entre el chorro y la ambiental se utiliza un termostato de inmersión JP Selecta, además de un secador de aire ya que el dispositivo no tiene protección contra la humedad. Se llegan a diferencias de temperatura de $4 \mathrm{~K}$.

El siguiente paso antes de realizar las mediciones es ajustar el cero del micrómetro Mitutoyo en el punto de contacto con la placa, el cual debe ser un el centro de la matriz de 8x8 sensores de medición, no en el centro geométrico, porque se tendrían valores desplazados y los programas de procesamiento de datos no tendrían resultados reales. A partir de este cero se hacen las mediciones de $\mathrm{H}$. En la figura 6 se presentan los dos esquemas del montaje, con la especificación de las cotas correspondientes a H y D.
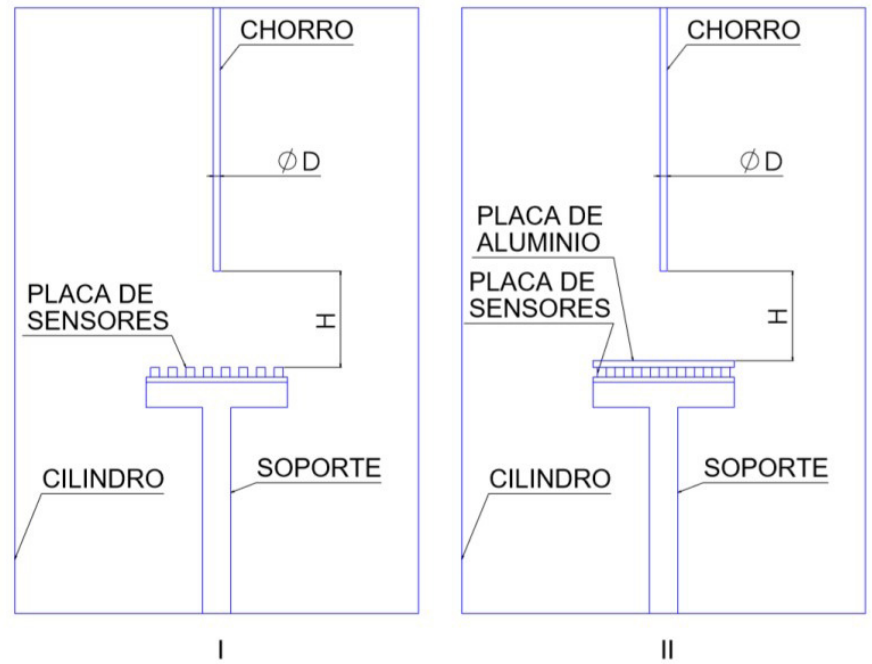

Figura 6. Esquema del montaje sin placa de aluminio (I), con placa de aluminio (II). 


\section{Resultados}

Los resultados de las visualizaciones se pueden observar en la figura 7, los cuales se obtuvieron con cámaras de alta velocidad y con la introducción de una emulsión de aceite y aire fresco, para las relaciones de $H / D$ limites $(H / D=1, H / D=5)$, se puede observar que ambos flujos están en régimen laminar después del impacto para la configuración del montaje II (Figura 6). En el caso $H / D=1$ se observa más concentración de aire en la placa ya que la distancia de impacto es menor, mientras que en para $\mathrm{H} / \mathrm{D}=5$ la visualización del impacto muestra una mayor dispersión al abrirse el chorro antes del impacto. También se observan eventuales patrones de turbulencia a medida que nos alejamos de la zona de impacto, especialmente para $H / D=5$.
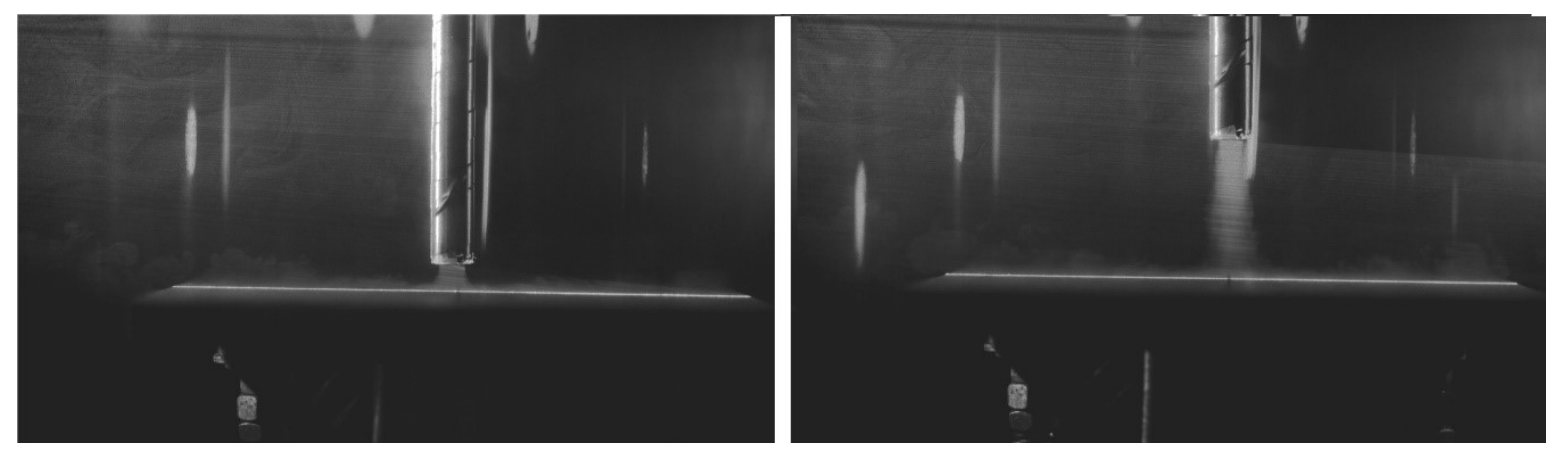

Figura 7. Visualizaciones del comportamiento del chorro a $H / D=1$ y $H / D=5$.

Las primeras mediciones se realizaron sin la placa de aluminio, con el fin de obtener la simetría en las mediciones de temperatura desde el punto de impacto (similares a anillos concéntricos) y comprobar la funcionalidad de la placa. En la figura 8 se muestran los anillos concéntricos de temperatura para relaciones de $H / D=1,2,3,4,5$ y un $\Delta T=4 \mathrm{~K}$. La información brindada por la placa de datos, gracias al uso de Labview se registra en un archivo de texto que después es procesado y graficado con la utilización de Matlab.

Debido al diseño de la placa, el efecto película no es visible, ya que los sensores están por encima de la placa. En los casos donde el chorro está más cerca de la placa las temperaturas frías están más concentradas en el centro. Sin embargo, se observa en la figura 8 que la diferencia entre gráficas es muy pequeña. Estos datos corresponden al montaje I de la figura 6.

Cuando se hacen las mismas mediciones para las mismas relaciones de $H / D$, pero agregando la placa de aluminio sobre los sensores (montaje tipo II de la figura 6), el efecto película aparece y hace que las dianas se vean invertidas, estando la parte más caliente en centro y las más frías en los extremos. Las velocidades en las zonas de impacto son casi cero, por lo tanto, la transferencia de calor por convección en esas zonas es muy baja y se obtienen los patrones que se muestran en la figura 9. Para esta figura, al igual que en la figura 8 , el procesado y graficado de los datos se hizo gracias a Matlab, el cual se inicia con un archivo de texto que es generado por Labview, definido por los creadores de la placa. 

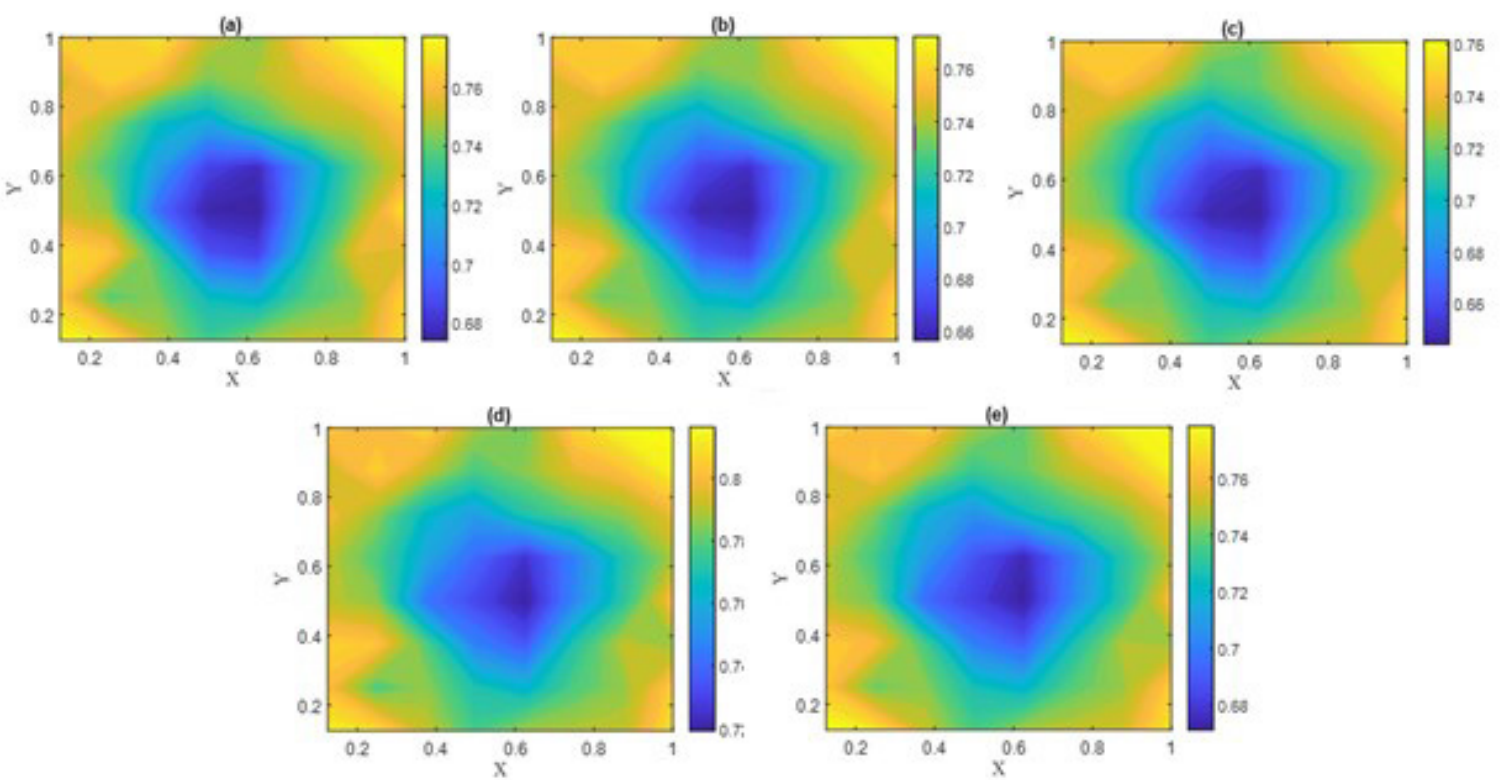

Figura 8. Anillos concéntricos de temperatura para un $\Delta T=4^{\circ} \mathrm{C}, H / D=1$ (a), $H / D=2$ (b), H/ $\mathrm{D}=3$ (c), $H / D=4$ (d), H/D=5 (e). En la configuración I. [Fuente propia. Matlab 2018]

Para cada relación de $\mathrm{H} / \mathrm{D}$, se obtiene una curva característica de temperatura promedio entre temperatura inicial en función de un tiempo característico $t_{0}$, este se conoce con la siguiente fórmula:

$$
e p c_{p} \frac{d \bar{T}}{d t}=h \bar{T}-T_{2}
$$

Además, teniendo en cuenta que

$$
\theta=\frac{\bar{T}-T_{1}}{T_{2}-T_{1}} \quad ; \quad \theta_{0}=\frac{\overline{T_{t=0}}-T_{1}}{T_{2}-T_{1}}
$$

y despejando matemáticamente $h$ se obtiene:

$$
h=e p c_{p} m
$$

donde $m$ es la pendiente de la siguiente ecuación:

$$
\ln \frac{\theta}{\theta_{0}}=m \cdot T+a
$$

Este valor de m se encuentra con la línea de mejor ajuste para cada uno de los datos tomados experimentalmente que corresponden a la evolución temporal de la temperatura. 

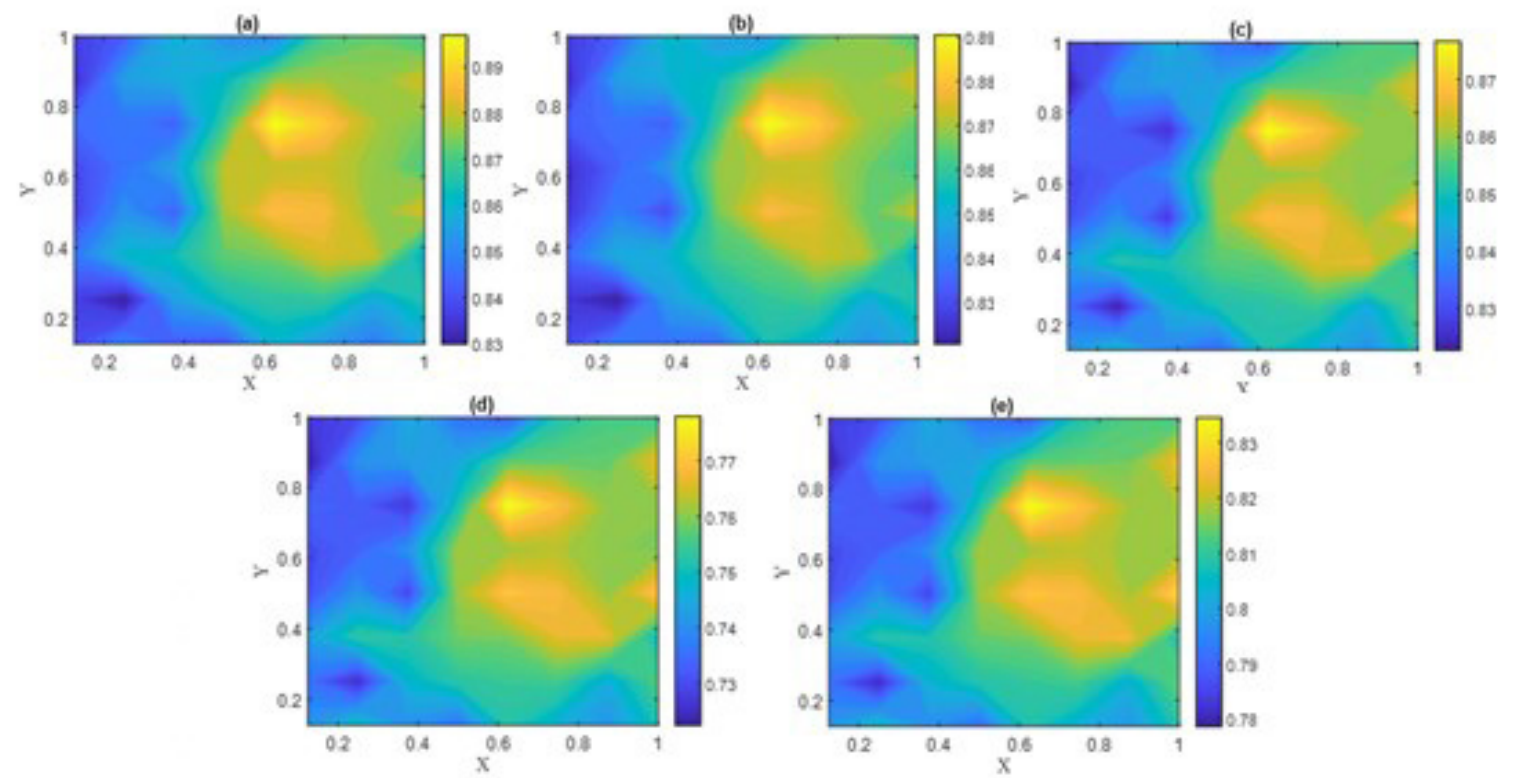

Figura 9. Anillos de temperatura para un $\Delta T=4 K, H / D=1$ (a), $H / D=2$ (b), $H / D=3$ (c), $H /$ $\mathrm{D}=4$ (d), $H / D=5$ (e). En la configuración II. [Fuente propia. Matlab 2018]

En la figura 10 se presentan las líneas de mejor ajuste (las líneas continuas) y los valores experimentales (diferentes formas). La dispersión entre los datos para una misma relación H/D es mínima lo que da certeza de la precisión en las mediciones, garantizando la bondad de los datos experimentales.

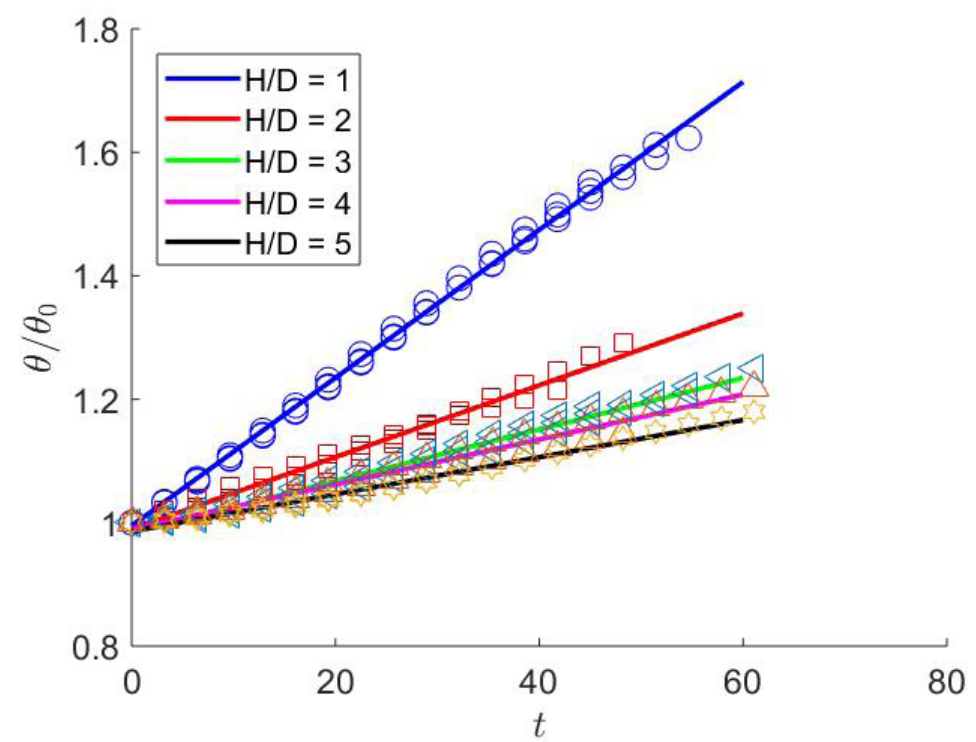

Figura 10. Relaciones de en función del tiempo adimensional t para las diferentes relaciones de H/D. [Fuente propia. Matlab 2018] 
Tomando los valores de densidad del aluminio laminado y el calor especifico del aluminio del libro de Transferencia de Calor, tabla A-2 [9] se calculan los valores de h para cada experimento, se obtiene un valor promedio por relación de H/D. El resultado se muestra en la figura 11.

Según la teoría los valores de h, para relaciones de gas-sólido en convección forzada no podían pasar de $250 \mathrm{~W} / \mathrm{m} 2 \mathrm{~K}$ y no podía ser menor a $2 \mathrm{~W} / \mathrm{m} 2 \mathrm{~K}$, los valores obtenidos en el modelo experimental rondan un rango de 60 a $220 \mathrm{~W} / \mathrm{m} 2 \mathrm{~K}$, por lo que se considera exitoso, tanto el experimento como el funcionamiento del dispositivo electrónico.

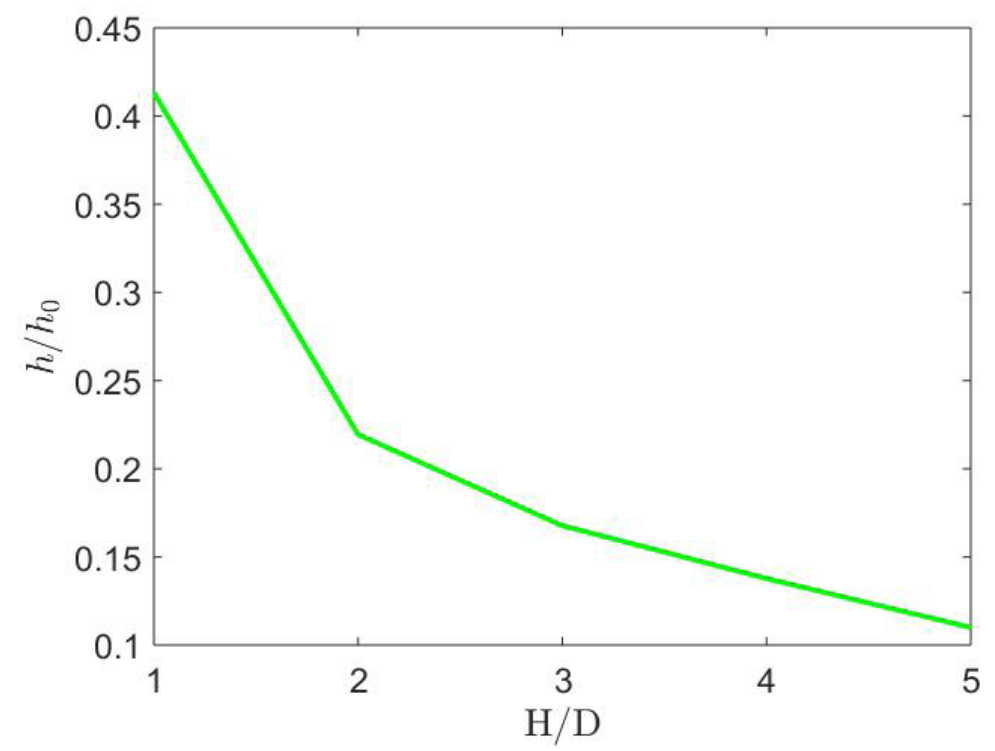

Figura 11. Valores de h/hO contra relaciones de H/D. [Fuente propia. Matlab 2018]

\section{Conclusiones y recomendaciones futuras}

Se ha montado un experimento para ensayar una placa de sensores de temperatura mediante una metodología electrónica de última generación, la cual dio resultados satisfactorios ya que se han encontrado valores de coeficiente de convección forzada en un rango de 60 a 220] W/m2 K, el cual está dentro de lo aceptado por la literatura. Además, las mediciones del dispositivo sin la placa de aluminio concuerdan con lo esperado y descrito en la teoría. Para relaciones mayores a $\mathrm{H} / \mathrm{D}=5$ los resultados no presentan variaciones significativas, de $\mathrm{H} / \mathrm{D}=1 \mathrm{a}$ $H / D=2$ es donde se produce la mayor disminución de $h$, probablemente asociado a un cambio de régimen en la zona de impacto. Las desviaciones entre los resultados para un mismo H/D permiten afirmar que los resultados son precisos y repetibles. Se estima oportuno relacionar en futuros trabajos los patrones de las visualizaciones (y el régimen laminar o turbulento del flujo) con el coeficiente h calculado, así como aumentar el rango de diferencia de temperaturas a valores superiores a $4 \mathrm{~K}$.

\section{Agradecimientos}

Se agradece profundamente a Sergio Pinazo y Alexandro Díaz por la ayuda brindada en el montaje experimental, a Raquel Fernández, José Hidalgo, Jorge Romero, José Martín y Francisco Ríos por permitir la utilización del dispositivo electrónico de medición. A la Universidad de Málaga (UMA) y al Tecnológico de Costa Rica (TEC) por la ayuda recibida para la estancia de movilidad, las instalaciones y los medios facilitados. 


\section{Referencias}

[1] P. G. Castillo, Estudio experimental del impacto sobre una superficie plana de un chorro frío, Málaga, España: Universidad de Málaga, 2011.

[2] J. M. Bergthorson, K. Sone, T. W. Mattner, P. E. Dimotakis, D. G. Goodwin y D. I. Meiron, Impinging laminar jets as moderate Reynolds numbres and separation distances, Pasadena, California 91125, USA: California Institute of Technology, 2005.

[3] J.-J. Shu y G. Wilks, Heat Transfer in the Flow of a Cold, Axisymmetric Vertical Liquid Jet Against a Hot, Horizontal Plate, Singapore: School of Mechanical and Aerospace Engineering, 1996.

[4] J. Baonga, H. Louahila-Gualous y M. Imbert, Experimental study of the hydrodynamic and a heat transfer of free liquid jet impinging a flat circular heated disk, Belfort, France: Femto ST, 2005.

[5] J. A. Higalgo Lopez, J. Romero Sanchez, R. Fernandez Ramos, J. Mantín Canales y J. Ríos Gómez, A lowcost, high-accuracy temperature sensor array, Málaga, Spain: Departamento de Electrónica, Universidad de Málaga, 2018.

[6] Liu, Chang, Cascioli, Heusch y McCarthy, Design and development of a thermal imaging system based on a temperature sensor array for temperature mesaurements of enclosed surfaces ans its use at the body-seat interface, California, USA: Measurement, 2017.

[7] R. Bardera Mora, Aplicación del PIV a la medida del coeficiente de resistencia aerodinámica, Madrid, España: Facultad de Ciencias Físicas, 2005.

[8] J. G. Marroquín, Determinación experimental de coeficientes de transferencia de calor para convección libre y forzada, Nuevo Leon: Nicolas de los Garza, 1998.

[9] Y. A. Cengel, Transferencia de Calor, México DF: McGraw-Hill, 2004.

[10] J. Coberan y R. Royo, «Mécanica de Fluidos,» Universidad Politécnica de Valéncia, 16 Junio 2006. [En línea]. Available: http://www.upv.es/ul/U0296621.pdf. [Último acceso: 1 Junio 2018]. 